\title{
Reliability research of vehicle electric window control system based on CAN-bus
}

\author{
Wansong Liu $^{\# 1}$, Wenjun Xiao ${ }^{\# 2}$, Linhu Hui ${ }^{\# 3}$, Yi Wang * \\ \# School of Physics \& Electronics Science, Guizhou Normal University \\ 116 North Baoshang Road, Guiyang, 550001, China \\ * Corresponding author ,Key Laboratory of Automotive Electronics Technology of Education \\ Department of Guizhou Province
}

\begin{abstract}
In recent years, the vehicle electric window control system based on CAN-Bus has been widely used, but its reliability research is insufficient. In this paper, factors that influence the reliability of the electric window control system had been qualitatively analyzed, and the failure rate of window node had been quantitatively analyzed. Then the reliability of 3 kinds of vehicle electric anti-pinch window control algorithm had been studied, and based on that, corresponding solutions had been given to solve the problems which influence the reliability of the vehicle CAN-bus electric window control system, through that, the reliability of the vehicle CAN-bus electric window control system had been improved .
\end{abstract}

Keyword: Electric Window; CAN-bus; Anti-pinch algorithm; reliability

\section{INTRODUCTION}

Vehicle now become an indispensable means of transport in modern life. As more and more people could afford the car, they become to have a higher demand about car on safety, comfort and reliability. CAN bus is now widely used in automotive electric window control system [1-2], which improves the controllability of the car, creates a more comfortable driving environment and reduces the labor intensity of the driver and passengers. But it also brings much stricter requirements about the reliability of vehicle CAN-bus electric window control system. The reliability research of vehicle electric window control system based on CAN-bus is insufficient domestically. In reality, there are often car accidents due to the electric windows, which affect the normal running of the vehicle ${ }^{[3-4]}$.

\section{The Components of Vehicle CAN-bus Electric Window Control System}

An vehicle CAN-bus electric window control system is always composed of a window glass, an elevator, a DC motor, an elevator switch controller and so on, just as showed in Figure 1. In order to coordinate to the vehicle bodies, windows of modern car are basically adopted by large curvature curved

This work was supported by the National Natural Science Foundation of China (Grant No. 61262007, 61462015), by International Science \& Technology Cooperation Research Foundation of Guizhou Province (Grant No. [2014]7007), by Science and Technology Foundation of Guizhou province(Grant No.[2013]2228),by Course Team of SCM principle and application of Guizhou Normal Univercity(Grant No.KT2014007H), in part by Key Laboratory of Education Department of Guizhou Province (Grant No. KY word [2014] 213). glasses. The elevator is usually rope, gear or flexible arm type. DC motor is the key part of electric window elevator, and usually adopts reversible permanent magnet motor with inner decelerator. The speed of revolution could be reduced by worm gear reducer when the motor running. The compact-structure worm gear reducer has large reduction ratio and works smoothly without noise. When the helix angle is less than the friction angle, it could effectively prevent the reverse of the window glass. The window drive switch controller adopts the PIC18F258 microcontroller produced by Microchip within the vehicle temperature work range and high cost-performance ratio. On the PIC18F258 microcontroller, a CAN controller had been integrated, and that could simplify the system hardware design and improve the reliability of the system.

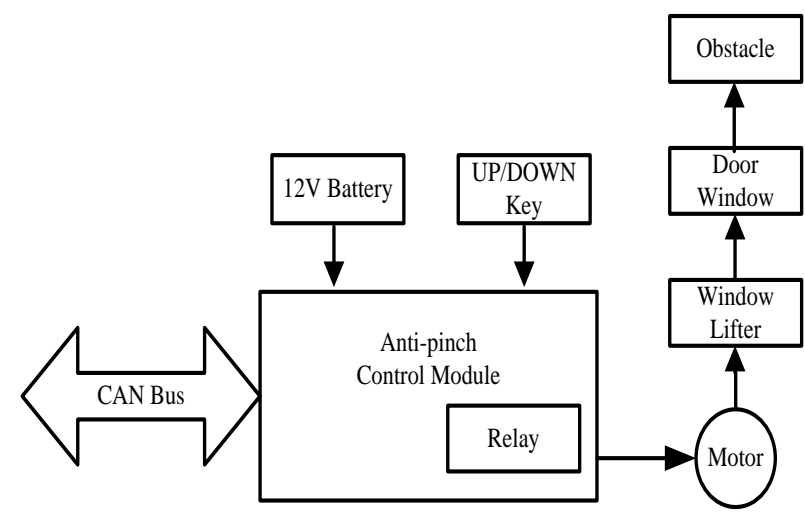

Fig.1 Structure of vehicle electric window control system

The communication network of electric window control system is consists of 4 CAN electronic controller nodes: the left front door main-controller, right front door sub-controller, left back door sub-controller and right back door subcontroller. These 4 CAN electronic controller nodes could communicate with each other through CAN-bus ${ }^{[5]}$.

The left front door main-controller could not only control the window glass of driver's side, but also could control the lift of the window glasses of the rest passengers' sides. Meanwhile, the window glasses of passengers could be separately controlled by each sub-controller. The main-controller is mainly composed of three parts, such as PIC18F258 microcontroller, 6N137 high speed optical coupler, PCA82C250 bus transceiver. Considering the design cost and software programming convenience, the sub-controllers select 
the same chip as the main-controller, and have the same hardware circuit structure.

PIC18F258 is a high performance single chip of Microchip, which has the characteristics of ultra-compact, low power consumption, low cost, and multi-variety. It has a wide range of applications. PIC18F258 microcontroller is the core part of the CAN-bus interface circuit to insure the smooth communication, and mainly used to not only send and receive data on the CAN-bus, but also realize the serial data encoding and decoding.

PCA82C250 is the CAN bus interface chip produced by Philips, and it is the interface between CAN controller and physical bus. It provides the ability to differential transmit and receive data on bus and fully compatible with ISO11898 standard. There are 3 kinds of different working methods, such as high speed, slope control and stand-by, while we choice the high speed method in this design. PCA82C250 has few pins and easy to use.

6N137 is the high-speed logic gate output optical coupler produced by Instrument General Company. Its maximum transmission delay time is $75 \mathrm{~ns}$ and the typical value is $46 \mathrm{~ns}$. The 6N137 high-speed optical coupler circuit could achieve the electrical isolation between nodes commendably, and improve the system's anti-interference ability and the ability to transmit signals. The vehicle electric window control system designed in this paper has flow advantages: simple circuit structure, easy to achieve, stable and reliable performance, and cost saving under meeting the performance requirements as far as possible.

III. Reliability analysis of the vehicle electric window control system

Reliability of the CAN bus automotive electric window control system refers to the ability of the system to complete the specified tasks under specified conditions within the prescribed time. For different electronic products, due to their different geometry, electrical property and other product characteristics, their reliability are different. When products lose their specified functions, whether it could be repaired, collectively referred to as failure. According to the relevant statistics, about $65 \%$ repairs of the vehicle electric window control system is due to electronic components failure. To improve the reliability of the vehicle electric window control system, could not only reduce the cost of products in entire life cycle and improve the utilization of products, but also prevent the occurrence of accidents and protect the users.

In order to analyze the reliability of the CAN bus vehicle electric window control system, the system should be divided into the reliability prediction units, and base on that to establish the reliability prediction model. The reliability prediction units are series connected, and each of them is independent. The failure rate of the whole system is taken by the minimum failure rate of each reliability prediction unit. Furthermore, the system mean time to failure and other reliability data could be obtained based on the failure rate of the whole system ${ }^{[6]}$.

According to the relevant knowledge, the failure rate of the microprocessor is:

$$
\lambda_{P}=\pi_{Q}\left[C_{1} \pi_{Y} \pi_{V}+\left(C_{2}+C_{3}\right) \pi_{E}\right] \pi_{L}
$$

The vehicle electric window control system is composed of three parts: motor drive module, CAN-bus communication module and MCU module. The MCU module is adopt by PIC18F258, and its reliability could be estimated by the method of stress analysis prediction:

$\pi_{E}=6.3, \pi_{Q}=0.5, \pi_{L}=1.0, \pi_{Y}=1.75, C_{1}=0.6, C_{2}=0.05$,

$C_{3}=0.12$, and $\pi_{V}=1.0$.

Its failure rate could be calculated by the formula:

$$
\lambda_{P}=\pi_{Q}\left[C_{1} \pi_{Y} \pi_{V}+\left(C_{2}+C_{3}\right) \pi_{E}\right] \pi_{L}=1.08 \times 10^{6}
$$

The calculated result of the failure rate shows that, for the normal use of PIC18F258 in the window node, the mean time to failure is 104 years.

PCA82C250 is the interface between the CAN controller and the physical bus, which mainly provides the ability to differential transmit and receive data on the bus. For the PCA82C250 chip, there are the following parameters:

$$
\begin{aligned}
& \pi_{E}=6.3, \pi_{Q}=0.5, \pi_{L}=1.0, \pi_{Y}=0.77, C_{1}=0.106, C_{2}=0.004, \\
& C_{3}=0.04, \text { and } \pi_{V}=1.0 .
\end{aligned}
$$

Its failure rate could be calculated by the formula:

$$
\lambda_{P}=\pi_{Q}\left[C_{1} \pi_{Y} \pi_{V}+\left(C_{2}+C_{3}\right) \pi_{E}\right] \pi_{L}=0.18 \times 10^{6}
$$

PIC18F258 MCU is integrated with CAN controller, and therefore CAN network can be set up to achieve CAN communication. Furthermore, its internal has PWM outputs, an ADC of 12 bit accuracy and a CPU up to $40 \mathrm{MHz}$. meanwhile, it support 32 interrupt and reset sources.

Based on the results of each predicted unit abovementioned, the whole failure rate of CAN communication module could be estimated:

$$
\lambda_{P}=\min \left\{1.08 \times 10^{6}, 0.18 \times 10^{6}\right\}=0.18 \times 10^{6}
$$

Using the same method, result could be get that the mean time to failure of CAN bus communication system in normal use is about 20 years, and it could meet the vehicle operating requirements. Based on the failure rate analysis of the electronic control unit, it is known that the failure rate of CAN controller PCA82C250 chip accounted for the main factor in the entire system failure rate. In order to improve the system reliability, high grade of CAN controller chips could be chosen.

IV. Reliability analysis of vehicle electric window anti-pinch control algorithm

The anti-pinch function of the vehicle electric window is realized by algorithm, such as the amplitude method, the slope method, the integral method. The main problems to be solved by Anti-pinch algorithm are as flow: (1) region detected: $4 \mathrm{~mm}$ 
to 200mm; (2) maximum clamping force: $100 \mathrm{~N}$; (3) motor reverse when clamping object; (4) deflection angle test: $5 \mathrm{~N} / \mathrm{mm}$ to $20 \mathrm{~N} / \mathrm{mm}$. In addition, there should be an adaptive process to eliminate the impact on anti-pinch algorithm from the following two aspects: (1) mechanical and electrical parameters of the vehicle electric window would change (aging, deformation, wear, etc.) with the use of time; (2) the friction force would change due to environmental changes (temperature, humidity, freezing, etc.).

The anti-pinch function of the vehicle electric window is only working in the automatic rising process of the window, and in other cases (automatic descent, manual rise, and manual drop), there is no need to prevent clip. Therefore, it is necessary to get the relationship between the thrust force and the position of the window glass in the rising process of the vehicle electric window. Based on this principle, the algorithms are as flow:

\section{(1) Current and hall pulse discrimination method}

Firstly, detect the motor current; secondly, determine the initial force of the vehicle electric window. Then the relationship between the anti-pinch force and window motor current could be calculated. And the size of the clamping force could be got by detecting changes of the motor current when the vehicle electric window works. Hall pulse used to estimate whether the position of the window glass is in the range of anti-pinch or not, and detect the window glass is rising to the top or down in the end. Using this method, the size of the clamping force could be calculated by detecting the mean value and the maximum value of the motor current. Meanwhile, running speed and acceleration of the vehicle electric window could be calculated through the hall pulse, and the running status of the vehicle electric window could also be determined. The system block diagram is shown in Figure 2.

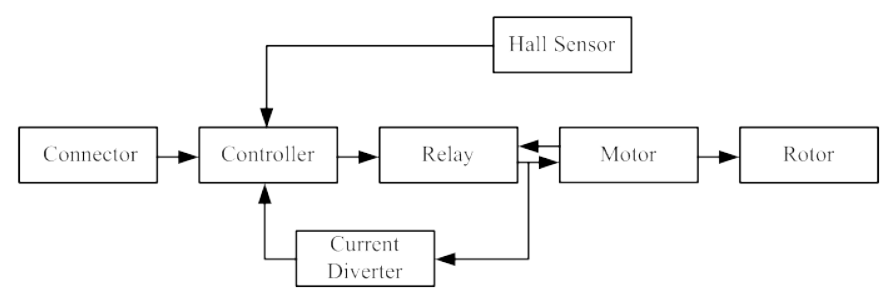

Fig.2 Block diagram of current and hall generator discrimination anti-pinch

$$
\text { system }
$$

\section{(2) Current ripple detection method}

Alternating current ripple would be generated when the window motor works, therefore the motor state can be detected according to the changes of the motor current ripple. Assuming the number of commutator bar in the motor is $n$, the current ripple frequency is $n$ times the motor speed $m$, so the ripple frequency is $f=n \times m$. After digital processing, the current ripple can be converted into pulse wave, which is similar to the hall pulse. The system block diagram is shown in Figure 3.

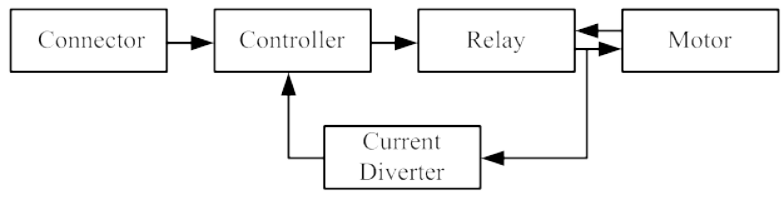

Fig.3 The block diagram of the current ripple detection algorithm.

V. Motor protect function of vehicle electric window control system

Due to the window motor is used frequently, the motor protection circuit is needed to ensure the reliability of the motor, which is as shown in Figure 4.

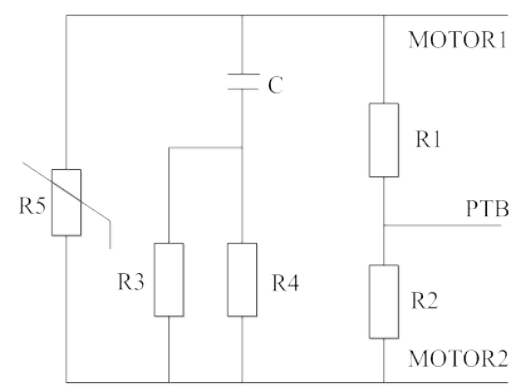

Fig.4 The motor protection circuit

PTB is a universal I/O port, which could be used not only as a bidirectional $\mathrm{I} / \mathrm{O}$ port, but also as an input port of $\mathrm{A} / \mathrm{D}$ converter; MOTOR1 and MOTOR2 are motor access interfaces; R1 and R2 are the same resistance; the terminal voltage of the PTB is half of the motor armature voltage and could be used to detect the motor armature voltage; resistor R3, $\mathrm{R} 4$ and capacitor $\mathrm{C}$ have formed a motor buffer circuit, which could absorb the oversized du/dt and di/dt; R5 is a pressure sensitive resistance, which would be short circuited to eliminate the sudden spike voltage and protect the motor when the output voltage of the relay is higher than the predetermined value.

VI. Fault self-diagnosis protect function of the vehicle electric window

The fault self-diagnosis protect function of the vehicle electric window plays an important role in improving the reliability of the whole vehicle electric window control system [7]. The vehicle electric window control system with selfdiagnosis protect function could judge the work status automatically, and timely implement the corresponding measures to avoid damage.

The vehicle electric window fault self-diagnosis system is realized through the VNH2SP30 chip. VNH2SP30 is a full bridge motor driver IC integrated with two single chip highside driver and two low-side switch. $\mathrm{EN}_{\mathrm{A}} / \mathrm{DIAG}_{\mathrm{A}}$ and $E_{B} / D_{A A G}$ are the fault self-diagnosis pins of the chip. According to the status of the port $I_{A}$ and $I N_{B}$, and collecting the voltages of $\mathrm{EN}_{\mathrm{A}} / \mathrm{DIAG}_{\mathrm{A}}$ and $\mathrm{EN}_{\mathrm{B}} / \mathrm{DIAG}_{\mathrm{B}}$ through $\mathrm{AD}$ chip, combing of the information to diagnose the fault of the vehicle electric window. The workflow is shown in Figure 5. 


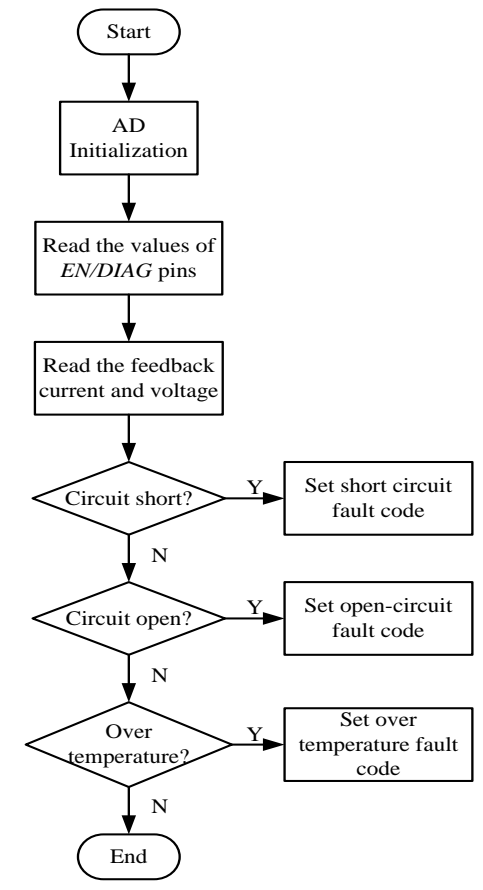

Fig. 5 the flow chart of self-diagnosis protect function

\section{Adaptive function}

Vehicle work environment is very harsh, often in high temperature, vibration, dust and other conditions. The mechanical parts of vehicle electric window control system would be wore with the extension use of time, which greatly affects the stability of the vehicle electric window. Adaptive function could cope with the impact of various environmental changes, and improve the stability and robustness of the vehicle electric window ${ }^{[7]}$. The working process is: when the window glass rises to the top, if the difference between current and last hall pulse value is larger, abandon the current pulse value and save the last hall pulse value to the register; if the difference between current and last hall pulse value is small, save the current hall pulse value to the register and update the position data of the top anti-pinch point and the top soft-stop point, then recycle the process.

\section{VIII.Conclusion}

The reliability of vehicle CAN-bus electric window control system plays an important role in the safety of vehicle driving In this paper, the reliability of the vehicle CAN bus electric window system had been analyzed, and its deficiencies in communication system, anti-pinch algorithm, window motor, fault diagnosis and adaptive system had been pointed out, then the corresponding reliability improvements in those aspects had been put forward. Reliability research could not only ensure the reliability of the vehicle electric window and the safety of users, but also reduce the cost of vehicle maintenance caused by the failure of the electric window. It is believed that with the popularization of the vehicle electric window technology, its reliability research would be further developed in the future.

\section{References}

[1] WANG Liming, XIA Li, SHAO Ying. Design and application of CAN field bus system [M]. Beijing: Electronic Industry Press, 2008.

[2]Xiao Zhaoxia. Hardware Design of Vehicle Door with Local Interconnect Network Bus. IEEE International conference On Computer Design and Application, 2011.

[3] LIAO Le-ping,YANG Song,GAO Shen,et al.The design of body control system based on SAEJ1939[T].Xia men: Xia Men University, 2011.

[4] XIE You-hua, YIN Cheng-liang, SHU Jie. Application of LIN bus in the control of electric window system [J].micro computer information, 2008, 24(5):223-224

[5] Daiqiang Wang, Shiyou Gao, Yuqing Chen. Intelligent Control System Based on CAN-bus For Car Doors and Windows [J] Proceedings of the 3rd international conference on Anti-Counterfeiting, security, and identification in communication, IEEE Press Piscataway, 2009.

[6] Zhu Shuanghua. Fault diagnosis and detection technology of vehicle CAN-bus system [M]. Changsha: National University of Defense Technology press, 2008.11

[7] Tom Denton. Vehicle electrical and electric system [M].BeiJing: China Machine Press, 2008 